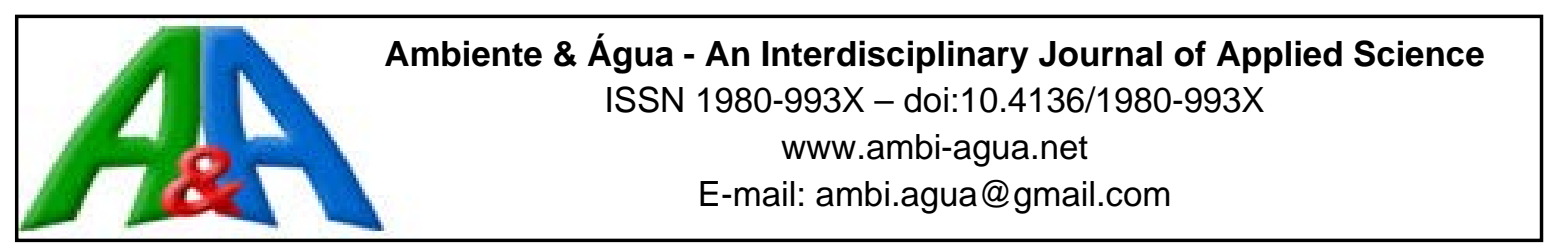

\title{
Influências edáfica e topográfica sobre a comunidade de palmeiras da floresta ombrófila densa Montana, Parque Estadual da Serra do Mar- SP, Brasil
}

\author{
doi:10.4136/ambi-agua.1921
}

Received: 12 Jun. 2016; Accepted: 11 Sep. 2016

\author{
Adriana Cristina Rosa Saraiva (in memoriam)'; \\ Simey Thury Vieira Fisch ${ }^{1 *}$; Maria Cecilia Barbosa Toledo ${ }^{1}$ \\ ${ }^{1}$ Universidade de Taubaté (UNITAU), Taubaté, SP, Brasil \\ Programa de Pós-Graduação em Ciências Ambientais \\ *Autor correspondente: e-mail: simey.fisch@gmail.com, \\ mceciliabt@gmail.com
}

\section{RESUMO}

O trabalho foi realizado na Floresta Ombrófila Densa Montana, situada entre $23^{\circ} 17^{\prime}$ a $23^{\circ} 24^{\prime} \mathrm{S}$ e $45^{\circ} 03^{\prime}$ a $45^{\circ} 11^{\prime} \mathrm{W}$, com o objetivo de avaliar a influência das variações microambientais do solo, da serapilheira e da topografia na composição e estrutura da comunidade de palmeiras. Em duas parcelas permanentes de um hectare, cada uma dividida em três transecções de $10 \times 100$ m e subparcelas de 10 x 10 m, foram registradas todas as palmeiras existentes com o propósito de conhecer a riqueza das espécies e estrutura das populações. Nas subparcelas, amostras de solo foram coletadas para a determinação das propriedades química e física do solo; a espessura de serapilheira foi determinada em cinco pontos aleatórios e a microtopografia de cada sub-pacela foi classificada em cume, vertente e vale. Foram amostrados 3.221 ind./0,6 ha e constatada a ocorrência de quatro espécies: Euterpe edulis Mart., Geonoma gamiova Barb. Rodr., G. pohliana Mart. e G. schottiana Mart. Em função das condições de relevo existentes no local os solos dominantes ao longo dos transectos são jovens do grande grupo Cambissolos caracterizados por apresentarem teores mais elevados de areia $(55,00 \%)$ e silte $(20,67 \%)$, menores de argila $(24,33 \%)$ e baixa fertilidade natural evidenciada pela excessiva acidez $(3,7)$, alta saturação em alumínio (82,3\%), saturação em bases muito baixa $(3,9 \%)$ e teores dos macronutrientes Ca $(2,1), \mathrm{Mg}(1,9), \mathrm{K}(1,1)\left(\mathrm{mmol}_{\mathrm{c} .} \mathrm{dm}^{-3}\right)$ e P $(4,9)$ $\left(\mathrm{mg} . \mathrm{dm}^{-3}\right)$ também muito baixos. As análises das toposequências demonstraram que a posição cume possui condições de fertilidade um pouco superiores às classes vertente e vale. A classe vale apresentou teores em areia superior às demais posições. Houve pequena diferença na espessura da camada de serapilheira com valores de 3,0 cm para o cume, 2,5 cm para a vertente e 2,3 cm para a posição vale. A heterogeneidade microambiental ocasionou variação na distribuição e composição de apenas algumas espécies de palmeiras, em especial espessura da camada de serapilheira e concentração de areia, que influenciaram a ocorrência de plântulas e jovens de G. gamiova.

Palavras-chave: arecaceae, Mata Atlântica, topografia. 


\title{
Edaphic and topographic influence on the palm community in a montane tropical rain forest, State Park of Serra do Mar-SP, Brazil
}

\begin{abstract}
The work was carried out in tropical montane rain forest, situated at $23^{\circ} 17^{\prime}-23^{\circ} 24^{\prime} \mathrm{S}$ and $45^{\circ} 03^{\prime}-45^{\circ} 11^{\prime} \mathrm{W}$, in order to access how the micro-environmental variations of soil, topography and litter affect the composition and structure of the palm community. In two permanent plots of one hectare, each divided into three transects $(10 \times 100 \mathrm{~m})$ and subplots $(10$ $\mathrm{x} 10 \mathrm{~m}$ ), all existing palms were registered in order to determine the richness of species and the population structure. Soil samples were collected in sub plots to determine the chemical and physical properties of the soil. The thickness of the litter was determined at five random points, and the microtopography of each one was classified into ridge, slope and valley. The study recorded 3221 individuals/0.6 ha and the occurrence of four palm species: Euterpe edulis, Geonoma gamiova, G. pohliana and G. schottiana. Due to the existing relief conditions at the location, the dominant soils throughout the transects are young soils of the great Cambisol group. These soils are characterized by higher content of sand (55.00\%) and silt (20.67\%), minor clay (24.33\%), low natural fertility evidenced by the excessive acidity (3.7), high aluminum saturation (82.3\%), very low base saturation (3.9\%) and also very low contents of the macronutrients $\mathrm{Ca}$ (2.1), Mg (1.9), K (1.1) (mmol $\mathrm{dm}^{-3}$ ) and P (4.9) (mg. dm ${ }^{-3}$ ). The analyses of toposequences showed that the peak position has fetility conditions slightly higher than those of the slope and valley classes. The valley class had higher sand content than the other positions. There was a slight difference in the litter layer thickness with values of $3.0 \mathrm{~cm}$ for the ridge, 2.5 $\mathrm{cm}$ for the slope and $2.3 \mathrm{~cm}$ for the valley position. The micro-environmental heterogeneity caused variation in the distribution and composition of only a few species of palms, especially litter layer thickness and sand concentration, which influenced the occurrence of G. gamiova seedlings and juveniles.
\end{abstract}

Keywords: arecaceae, Atlantic Rain Forest, soil, topography.

\section{INTRODUÇÃO}

Entre as diversas florestas dotadas de grande heterogeneidade de ambientes, destaca-se a Floresta Ombrófila Densa Atlântica ou como é popularmente chamada Mata Atlântica, formação florestal que se estendia de nordeste a sul na costa brasileira. Sua riqueza de espécies vegetais, decorrente da grande heterogeneidade de ambientes, deve-se a uma larga amplitude de variações micro-topográficas, altitudinais, latitudinais e climáticas que resulta em uma paisagem complexa (Morellato e Haddad, 2000; Scudeller et al., 2001; Scarano, 2002).

Em função da variação do relevo a Floresta Ombrófila Densa recebe diversas denominações, sendo a Floresta Ombrófila Densa Montana o tipo florestal que no sudeste brasileiro abrange altitudes entre 500 e 1.500 m aproximadamente (Veloso et al., 1991). Em especial as florestas montanas, em decorrência do gradiente de elevação, sofrem alterações causadas pela altitude e clima, que podem intervir na estrutura e distribuição das comunidades vegetais (Gentry, 1988). Entre essas alterações destacam-se a radiação solar, a água e os nutrientes, que interferem na distribuição das plantas e nos padrões de disponibilidade de recursos. Outra característica ocasionada pelo aumento da elevação é a exposição crescente à formação de nuvens e nevoeiros. Em conjunto estes fatores vão tornando as árvores gradualmente menores, seus troncos mais curvados e retorcidos e as palmeiras são substituídas frequentemente pelos bambus (Grubb, 1977; Kappelle et al., 1996; Richards, 1996; Resende et al., 2002). 
A microtopografia, juntamente com as propriedades físico-químicas do solo e a matéria orgânica, forma uma heterogeneidade ambiental que, interagindo nas comunidades de plantas, faz as respostas das espécies formarem locais com características próprias (Carvalho et al., 2005). Segundo Widyatmoko e Burgman (2006), as palmeiras são adaptadas às condições específicas de solos, tanto em relação à qualidade nutricional quanto à drenagem. As variações na umidade solo, devido às secas prolongadas, podem afetar fortemente a comunidade de palmeiras e gerar um aumento na sua mortalidade. A influência dos gradientes de drenagem na distribuição de determinados estádios ontogenéticos em palmeiras foi demonstrada para a espécie amazônica Euterpe precatoria Mart., cujos indivíduos adultos se restringem a solos encharcados, enquanto os juvenis ocorrem também em solos bem drenados (Kahn e Castro, 1985).

A quantidade de serapilheira ou liteira depositada sobre o chão da floresta varia ao longo de um intervalo de escalas espaciais e temporais e é determinada principalmente pelo clima, sazonalidade, topografia, materiais de origem do solo, e distribuição das espécies vegetais (Golley, 1983; Ross, 1998). Apesar disso, são poucos os estudos que relacionam a serapilheira à ocorrência das palmeiras. Entre esses estudos destacam-se os realizados na Amazônia por Cintra et al. (2005) e Sousa (2007), quando foi observada a influência da profundidade da serapilheira na composição da comunidade de palmeiras.

Estudos sobre diversos aspectos da ecologia florestal vêm sendo desenvolvidos principalmente com palmeiras da floresta amazônica e das montanhas andinas (Vormisto et al., 2004; Svenning et al., 2009; Eiserhardt et al., 2011). E mesmo sendo esse grupo de plantas representado pela espécie arbórea de maior abundância do bioma Mata Atlântica, a palmeira Euterpe edulis Mart. (Scudeller et al., 2001; Oliveira et al., 2014), são escassas as informações sobre de que forma a heterogeneidade ambiental, como solo, topografia e serapilheira, podem afetar a comunidade de palmeiras na Floresta Ombrófila Densa Montana.

O presente estudo procurou identificar de que forma as variações microambientais na Floresta Ombrófila Densa Montana do Núcleo Santa Virgínia, Parque Estadual da Serra do Mar, Estado de São Paulo, influenciam a ocorrência da comunidade de palmeiras. As variações ambientais foram estudadas ao longo de topossequências (cumes, vertentes e vales) buscando: 1. constatar de que forma causavam alterações na comunidade de palmeiras (riqueza e abundância), nas condições edáficas (aspectos físico-químicos) e no acúmulo de serapilheira e 2. compreender como essas variações bióticas e abióticas se correlacionam entre si.

\section{MATERIAL E MÉTODOS}

\subsection{Caracterização da área de estudo}

O estudo foi desenvolvido na Floresta Ombrófila Densa Montana, no Núcleo Santa Virgínia $23^{\circ} 17^{\prime}$ a $23^{\circ} 24^{\prime} \mathrm{S}$, e $45^{\circ} 03^{\prime}$ a $45^{\circ} 11^{\prime}$ W no Parque Estadual da Serra do Mar, São Luís do Paraitinga - SP. Os solos existentes na área são predominantemente da classe Cambissolo Háplico. Nas regiões de relevo forte ondulado encontra-se a associação da classe Cambissolo Háplico Distrófico com Latossolo Vermelho Amarelo Distrófico e em relevo montanhoso a presença de Neossolos Litólicos Distrófico (Embrapa, 2009).

O clima da região segundo classificação de Köppen é temperado úmido com inverno seco e verão temperado (Cwb). Os meses mais úmidos são dezembro, janeiro e fevereiro, a temperatura média anual é $18^{\circ} \mathrm{C}$ e o nível de precipitação média é de $2.180 \mathrm{~mm}$ ao ano, com estações seca no outono e inverno. 


\subsection{Caracterização da comunidade de palmeiras}

Foram alocadas duas parcelas permanentes de um hectare distantes entre si cerca de 800 m, denominadas de A e B, ao longo da trilha da Cachoeira da Fumaça, próximo à Base Itamambuca do Núcleo Santa Virgínia. O levantamento da comunidade de palmeiras foi realizado em três transecções de 10 x 100 m.parcela $^{-1}$ permanente divididas em subparcelas de 10 x 10m (Figura 1). As parcelas permanentes pertencem ao Projeto Biota Gradiente Funcional, aí correspondendo às parcelas $\mathrm{K}$ e $\mathrm{N}$, respectivamente $\mathrm{A}$ e $\mathrm{B}$ no presente trabalho, para mais detalhes veja Joly et al. (2012).

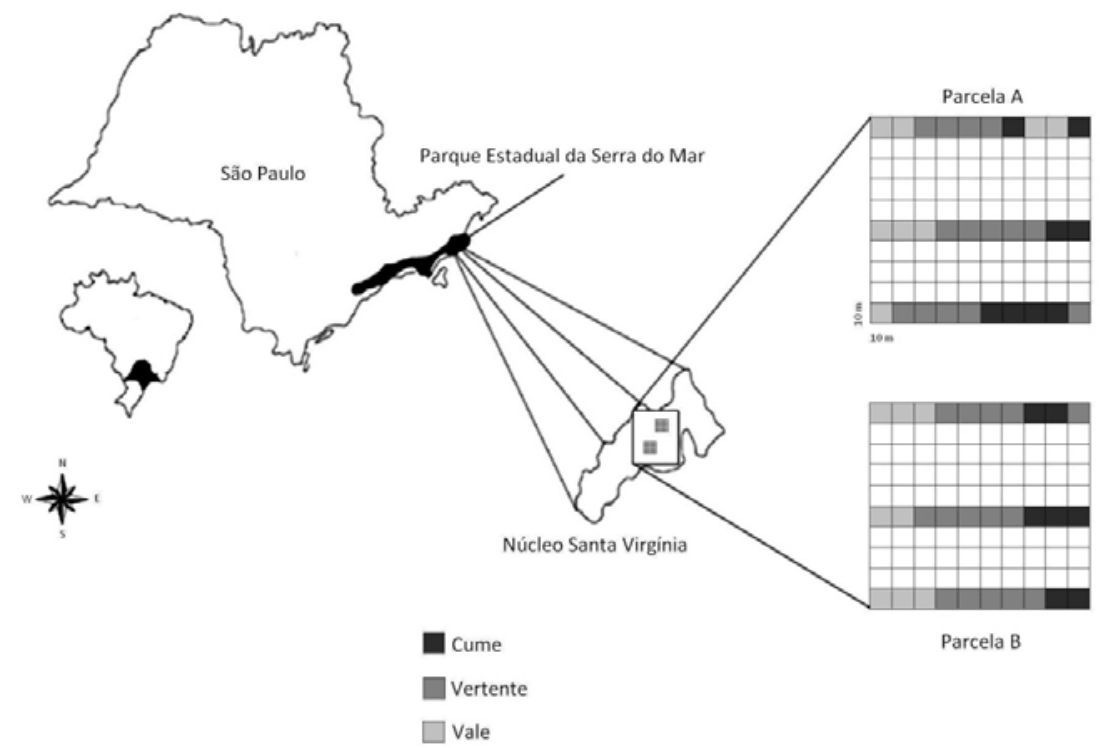

Figura 1. Localização do Núcleo Santa Virgínia, Parque Estadual da Serra do Mar- SP. As parcelas permanentes de um hectare estudadas foram divididas em transecções e as subparcelas foram classificadas em cume, vertente e vale.

Todas as palmeiras existentes em cada subparcela foram levantadas, desde plântulas a adultos, com o intuito de se conhecer a riqueza de espécies e formas de vida (arbustivas e arbóreas) que predominam neste ambiente. As palmeiras foram identificadas por comparação com duplicatas do material botânico coletado na região e depositado no herbário do Museu de Biologia Professor Mello Leitão (MBML). A partir da biometria completa (medidas de altura, diâmetro, número de folhas e folíolos e presença de semente aderida ao caule ou estruturas reprodutivas, inflorescências e infrutescências) e da avaliação no campo, os indivíduos de cada espécie foram classificados em três estádios ontogenéticos. Para tanto se adaptou as categorias propostas por Souza et al. (2003) para palmeiras do gênero Geonoma e Silva et al. (2009) para a espécie Euterpe edulis. Foram utilizados os seguintes critérios para classificação dos estádios: plântulas - indivíduos com semente aderida ao caule e/ou presença de folhas bífidas (Geonoma) ou palmadas (E. edulis), dependendo da espécie; jovens - indivíduos apresentando expansão do estipe e aumento na segmentação foliar; e adultos - indivíduos com sinais evidentes de floração e frutificação.

\subsection{Levantamento das variáveis ambientais}

Para classificar as variações microtopográficas da parcela A, com altitudes entre 1.049 e $1.071 \mathrm{~m}$, e da parcela B, com 1.014 a $1.027 \mathrm{~m}$ de altitude, seguiu-se a classificação utilizada por Yasuhiro et al. (2004). As subparcelas de cada transecção estudada foram classificadas como cume, vertente e vale. A classe cume correspondeu às subparcelas em que a altitude média foi maior que as demais na transecção. Por sua vez a classe vale apresentou os menores valores 
de altitude média na transecção em questão e a vertente os valores entre estes intervalos (Figura 1).

Para as análises químicas e físicas do solo $(\mathrm{pH}$, teores de $\mathrm{P}, \mathrm{K}, \mathrm{Ca}, \mathrm{Mg}, \mathrm{Al}, \mathrm{H}+\mathrm{Al}$; soma de bases - SB, saturação por bases - V\%, capacidade de troca catiônica - CTC, matéria orgânica - MO e teores de areia, silte e argila, umidade) foram coletadas amostras na camada superficial de 0-20 cm, em pontos aleatórios em cada uma das sessenta subparcelas. As amostras foram separadas de acordo com a classe de relevo do local de coleta (cume, vertente e vale), homogeneizadas e analisadas no Laboratório de Análise de Solos do Departamento de Ciências Agrárias da Universidade de Taubaté, SP utilizando a metodologia recomendada pela Embrapa (1997). A coleta dos solos se deu durante mês de julho de 2009, correspondente à estiagem de inverno.

A metodologia para análise da espessura da camada de serapilheira foi a mesma realizada por Portela e Santos (2007). Para essa medida foi utilizada uma régua de $30 \mathrm{~cm}$ em cinco pontos aleatórios (frente, atrás, direito, esquerdo e centro de cada subparcela), determinando-se em seguida a média da espessura da serapilheira.

\subsection{Análise estatística}

Para proceder às análises estatísticas, primeiramente foi aplicado o teste de normalidade dos dados dos estádios ontogenéticos das palmeiras e das variáveis ambientais. Uma vez que foi constatado que os dados não apresentavam distribuição normal, foi realizado a posteriori o teste não-paramétrico de Kruskal-Wallis para a comparação dos valores médios dos estádios ontogenéticos da comunidade de palmeiras nas parcelas A e B. Para comparações entre os valores médios do número de indivíduos de palmeiras e as diferentes classes topográficas foi utilizado o Teste U de Mann-Whitney.

As comparações da umidade do solo, classes topográficas e serapilheira das parcelas foram feitas por meio da análise de variância, seguidas de Teste de Tukey-Kramer. Os parâmetros edáficos foram analisados por meio do Teste $t$ não pareado e Teste de Wilcoxon, quando apropriado. As correlações entre a comunidade de palmeiras e as 16 variáveis ambientais foram determinadas pelo Teste de Spearman (Zar, 1996). Para conhecer os efeitos das variáveis nos adultos de E. edulis e $G$. gamiova e definir um modelo de distribuição foi realizado uma regressão múltipla usando as variáveis umidade do solo, classes topográficas, declividade e serapilheira das parcelas $\mathrm{R}^{2}$ e AIC de Akaike foram os indicadores usados para seleção do melhor modelo, para análise foi utilizado o programa XLSTAT 2016.v.03.

\section{RESULTADOS E DISCUSSÃO}

\subsection{Variáveis edafo-topográficas}

Os solos do trecho da Floresta Ombrófila Densa Montana estudado no Núcleo Santa Virgínia se caracterizam por apresentarem em média a classe textural franco argilo arenosa (Tabela 1), com a parcela B possuindo uma proporção em areia um pouco superior e de silte inferior em relação à parcela A. Ao longo de todas as classes topográficas a maior fração foi de areia, em especial nos vales. Além da concentração de areia, nas vertentes destaca-se presença significativa de silte (Tabela 1). Os teores elevados em areia e silte são característicos da classe Cambissolo (Embrapa, 2009) existente no local com influência também dos processos erosivos que ocorrem devido às condições de relevo da região.

Oliveira et al. (2014) relacionaram a maior presença da fração areia nos vales como resultante dos processos erosivos de transporte e acúmulo. Nas áreas mais planas do topo de encostas ocorrem maiores teores de silte e argila devido à ação de transformação dos minerais das rochas condicionadas pela maior taxa de infiltração da água e menores perdas pela ação erosiva. Resultados semelhantes foram encontrados em uma floresta semidecidual em Viçosa- 
MG, onde os solos arenosos ocorriam mais nas baixadas do que nos topos de morro (Martins et al., 2003).

Em função das ações dos processos erosivos condicionados pelo relevo, em geral os solos das baixadas se apresentaram com maior umidade em relação aos de topo, como observado em uma floresta semidecidual em Ingaí-MG (Brotel et al., 2002) e na floresta alto-montana em Bocaina de Minas-MG (Carvalho et al., 2005).

A camada de serapilheira na floresta montana variou de espessura entre as classes topográficas dentro das parcelas estudadas (Tabela 1). Quando se comparou as parcelas observou-se que na parcela A a camada de serapilheira foi cerca de $2 \mathrm{~cm}$ mais espessa em relação à parcela $\mathrm{B}$ e que as classes topográficas vale e vertente tiveram diferenças significativas, indicando haver variação na composição florística local, responsável pela produção do folhiço. Apesar dos testes estatísticos não acusarem diferenças entre as medidas em função da topografia, observou-se que as menores espessuras da camada de serapilheira ocorreram nos vales. O que se encontrou foi o contrário do que normalmente é esperado. Schlittler et al. (1993), estudando classes topográficas na Floresta Estacional Semidecidual (baixada, topo e vertente) em Paranapanema-SP, encontraram valores mais elevados de deposição de material orgânico na baixada, indicando que a topografia pode condicionar o acúmulo de serapilheira.

Tabela 1. Variáveis edáficas e topográficas das parcelas e variáveis químicas e texturais das amostras do solo superficial (0-20 cm de profundidade) coletadas nas 60 subparcelas das parcelas permanentes A e B na Floresta Ombrófila Densa Montana no Núcleo Santa Virgínia, SP. Os valores são médias e desvio padrão das amostras nas diferentes classes topográficas. As comparações foram feitas pelo teste $\mathrm{T}$ não-pareado $(\mathrm{P}<0,05)$ para as variáveis químicas e Teste de Wilcoxon para Ca e Mg nos vales das parcelas. Teste de Tukey- Kramer $(\mathrm{P}<0,05)$ para variáveis serapilheira e umidade do solo. ns: não significativo.

\begin{tabular}{|c|c|c|c|c|c|c|c|c|c|}
\hline \multirow[b]{2}{*}{ Variáveis } & \multicolumn{3}{|c|}{ Cume } & \multicolumn{3}{|c|}{ Vertente } & \multicolumn{3}{|c|}{ Vale } \\
\hline & $\begin{array}{c}\mathrm{A} \\
\mathrm{n}=8\end{array}$ & $\begin{array}{c}\mathrm{B} \\
\mathrm{n}=7\end{array}$ & $\mathrm{P}$ & $\begin{array}{c}\mathrm{A} \\
\mathrm{n}=14\end{array}$ & $\begin{array}{c}\mathrm{B} \\
\mathrm{n}=15\end{array}$ & $\mathrm{P}$ & $\begin{array}{c}A \\
n=8\end{array}$ & $\begin{array}{c}\mathrm{B} \\
\mathrm{n}=8\end{array}$ & $\mathrm{P}$ \\
\hline $\mathrm{pH}$ & $3,7( \pm 0,1)$ & $3.7( \pm 0,1)$ & ns & $3,6( \pm 0,1)$ & $3.7( \pm 0,05)$ & ns & $3,7( \pm 0,05)$ & $3.7( \pm 0,1)$ & ns \\
\hline $\mathrm{MO}\left(\mathrm{g} \mathrm{dm}^{-3}\right)$ & $69( \pm 17)$ & $38( \pm 11)$ & ns & $56( \pm 12)$ & $42( \pm 8)$ & ns & $46( \pm 14)$ & $48( \pm 3,5)$ & ns \\
\hline $\mathrm{P}\left(\mathrm{mg} \mathrm{dm} \mathrm{dm}^{-3}\right)$ & $4( \pm 1,7)$ & $5( \pm 1)$ & ns & $4,6( \pm 2)$ & $5( \pm 1)$ & ns & $6( \pm 3,5)$ & $5( \pm 4)$ & ns \\
\hline $\mathrm{K}^{+}\left(\mathrm{mmol}_{\mathrm{C}} \mathrm{dm}^{-3}\right)$ & $1,2( \pm 0,1)$ & $1( \pm 0,1)$ & ns & $1,1( \pm 0,3)$ & $1.2( \pm 0,3)$ & ns & $1( \pm 0,5)$ & $1.2( \pm 0,4)$ & ns \\
\hline $\mathrm{Ca}^{++}\left(\mathrm{mmol}_{\mathrm{C}} \mathrm{dm}^{-3}\right)$ & $3,6( \pm 4,6)$ & $1.3( \pm 0.6)$ & 0,031 & $2( \pm 1)$ & $2( \pm 1)$ & ns & $2( \pm 0)$ & $2( \pm 1,5)$ & ns \\
\hline $\mathrm{Mg}^{++}\left(\mathrm{mmol}_{\mathrm{c}} \mathrm{dm}^{-3}\right)$ & $3( \pm 2)$ & $1.3( \pm 0.6)$ & ns & $2(0,6)$ & $1.7( \pm 0,6)$ & ns & $2( \pm 0)$ & $1.7( \pm 0,6)$ & ns \\
\hline $\mathrm{H}^{+}+\mathrm{Al}^{+++}\left(\mathrm{mmol}_{\mathrm{c}} \mathrm{dm}^{-3}\right)$ & $131( \pm 16)$ & $109( \pm 30)$ & ns & $144( \pm 43)$ & $126( \pm 16)$ & ns & $145( \pm 18)$ & $134( \pm 20)$ & ns \\
\hline $\mathrm{Al}^{+++}\left(\mathrm{mmol}_{\mathrm{c}} \mathrm{dm}^{-3}\right)$ & $21( \pm 6)$ & $23( \pm 4)$ & 0,045 & $27( \pm 7,6)$ & $24( \pm 3)$ & ns & $25( \pm 4)$ & $25( \pm 3,4)$ & ns \\
\hline $\mathrm{SB}$ - soma de bases $\left(\mathrm{mmol}_{\mathrm{c}} \mathrm{dm}^{-3}\right)$ & $8 \pm 7)$ & $3.7( \pm 0,6)$ & 0,013 & $5,4( \pm 1,8)$ & $4.5( \pm 0,8)$ & ns & $5,1( \pm 0,8)$ & $5( \pm 2,3)$ & ns \\
\hline CTC $\left(\mathrm{mmol}_{\mathrm{C}} \mathrm{dm}^{-3}\right)$ & $139( \pm 21)$ & $113( \pm 30)$ & ns & $149( \pm 43)$ & $131( \pm 15)$ & ns & $150( \pm 18)$ & $139( \pm 19)$ & ns \\
\hline V - saturação por bases (\%) & $5,6( \pm 3,7)$ & $3.6( \pm 0,5)$ & ns & $3,6(1,5)$ & $3.6( \pm 1)$ & ns & $3( \pm 0,5)$ & $4( \pm 1,7)$ & ns \\
\hline Areia (\%) & $46( \pm 18)$ & $54( \pm 2)$ & 0,026 & $52( \pm 22)$ & $58( \pm 3,3)$ & 0,044 & $59( \pm 15)$ & $61( \pm 0,7)$ & 0,004 \\
\hline Silte (\%) & $25( \pm 9)$ & $20( \pm 1,4)$ & ns & $23( \pm 11)$ & $18( \pm 1)$ & 0,017 & $22( \pm 11)$ & $16( \pm 3)$ & ns \\
\hline Argila (\%) & $29( \pm 9)$ & $25( \pm 1,6)$ & ns & $24( \pm 12)$ & $24 \pm 3)$ & ns & $19( \pm 3,8)$ & $23( \pm 2,4)$ & ns \\
\hline Umidade (\%) & $32( \pm 8)$ & $25( \pm 6)$ & 0,029 & $36( \pm 10)$ & $21( \pm 3)$ & 0,000 & $37( \pm 19)$ & $24( \pm 6)$ & ns \\
\hline Serapilheira (cm) & $4( \pm 2)$ & $2( \pm 1)$ & ns & $3( \pm 1)$ & $2( \pm 1)$ & 0,005 & $3( \pm 1)$ & $1,6( \pm 0,5)$ & 0,020 \\
\hline
\end{tabular}


As parcelas A e B possuem solos fortemente ácidos, mantendo-se o $\mathrm{pH}$ praticamente constante ao longo das topossequências (Tabela 1). A matéria orgânica (MO) no solo foi encontrada em maior quantidade na parcela $A$ em relação à parcela $B$, justificada pelo maior acúmulo da serapilheira. O cume na parcela $\mathrm{A}$ foi à classe topográfica que apresentou a maior concentração de $\mathrm{MO}$, diferentemente da parcela $\mathrm{B}$, onde a maior presença de $\mathrm{MO}$ se deu no vale. Os solos das parcelas possuem fertilidade muito baixa caracterizada pela acidez muito alta ( $\mathrm{pH}<4,3)$, porcentagem de saturação em bases muito baixa $(\mathrm{V} \%<25,0)$ e os teores dos macronutrientes potássio, fósforo, cálcio e magnésio também baixos (Raij et al., 1996). Os teores em alumínio são muito elevados resultando em uma porcentagem de saturação em alumínio muito alta ( $\mathrm{m} \%>50 \%)$ que indiretamente contribui no aumento da acidez do solo (Raij et al., 1996). Os menores valores foram encontrados nos cumes, aumentando nas vertentes em direção aos vales das parcelas, onde apresentaram os maiores valores.

Em relação às variações microtopográficas analisadas, o cume foi à classe que apresentou resultados significativos com maiores valores de Ca e SB, quando comparada à vertente e ao vale das parcelas, devido às melhores condições de fertilidade condicionadas pela forma tipo patamar de relevo. O que se observou foi contrário do encontrado por Rodrigues et al. (2007), que tiveram uma diminuição de Ca e SB com o aumento da elevação na microtopografia, e por Silver et al. (1994) que encontraram maiores teores de Ca no vale de suas parcelas. Os resultados da saturação por bases aqui encontrados correspondem a uma condição de solo em geral muito pobre em nutrientes por apresentarem V\% médio de 3,9\%, o que representa uma condição de fertilidade muito baixa (Raij et al., 1996).

A variação nos teores de nutrientes e alumínio em relação às variações das toposequências é decorrente principalmente dos processos erosivos condicionados pelo relevo. Os cumes por apresentarem platôs e estarem menos sujeitos à ação erosiva demonstraram melhores condições de fertilidade e acúmulo de serapilheira. A tendência observada de acúmulo de alumínio nos vales pode estar relacionada, de acordo com Vashchenko et al. (2007), a declividade acentuada promover uma maior perda de bases pelo movimento de transporte e acúmulo ocasionada pela ação erosiva da água. Padrões diferenciados foram observados por Rodrigues et al. (2007), que também verificaram um aumento de $\mathrm{Al}$ com o aumento da altitude, e por Zueng-Sang et al. (1997), que observaram a maior concentração de Al nas vertentes, como ocorreu na parcela B.

\subsection{Distribuição das espécies}

Foram amostrados, de plântulas a adultos, 3.221 indivíduos de palmeiras em 0,6 ha. As palmeiras foram mais abundantes na parcela B (1.695 ind) apesar do menor número de espécies (Euterpe edulis Mart., Geonoma gamiova Barb.Rodr. e Geonoma pohliana Mart.), em relação à parcela $\mathrm{A}$ (1.526 ind.0,3 ha $\mathrm{h}^{-1}$ ), que por sua vez foi mais rica em espécies, apresentando uma espécie a mais (G. schottiana Mart.).

Na floresta Ombrófila Densa Montana no Núcleo Santa Virgínia ocorreram quatro espécies de palmeiras, sendo uma de porte arbóreo (Euterpe edulis) e as demais de pequeno porte, que são componentes do subosque florestal (Geonoma gamiova, G. pohliana e G. schottiana). De acordo com Toledo e Fisch (2006), para um gradiente de Floresta Atlântica próximo ao local do presente estudo, foram identificadas oito espécies de palmeiras entre as altitudes 100 e 200 $\mathrm{m}$, decrescendo para quatro espécies a $850 \mathrm{~m}$ de altitude. A mesma influência do gradiente de altitude da Serra do Mar na comunidade de palmeiras foi observada por Oliveira et al. (2014). Segundo Svenning et al. (2009), o declínio da diversidade de espécies de palmeiras com a elevação da altitude pode ser atribuído à características filogenéticas da família Arecaceae, como a pouca tolerância a baixas temperaturas das altitudes mais elevadas (Tomlinson, 2006). Essas diferenças são acentuadas pela latitude. Em menores latitudes, como na floresta de terrafirme da Amazônia Central, Khan e Castro (1985) encontraram 32 espécies de palmeiras em 1,2 ha e Cintra et al. (2005), 29 espécies em 2 ha. 
Na floresta montana estudada a palmeira E. edulis foi a espécie de maior ocorrência, seguida de G. gamiova e a G. pohliana. Bastos Neto e Fisch (2007), estudando áreas próximas às do presente estudo, na mesma unidade de conservação - Núcleo Santa Virgínia, porém no entorno de áreas de deslizamentos, também encontraram E. edulis e G. gamiova como as mais representativas, seguidas de G. pohliana. Em ambas as situações também foram observadas a ocorrência de outra espécie, G. schottiana, cuja abundância foi bem maior no entorno dos deslizamentos do que nas áreas preservadas aqui estudadas, o que pode indicar que a mesma se beneficia com as alterações de locais que sofreram perturbação.

Quanto à estrutura das populações das palmeiras, destaca-se a elevada ocorrência de plântulas da palmeira E. edulis, que representou uma abundância total de $83 \%$ dos indivíduos amostrados em relação aos jovens (12\%) e os adultos (5\%), nas duas parcelas (A e B). Quando se compara a distribuição dos estádios ontogenéticos das parcelas $\mathrm{A}$ e $\mathrm{B}$, observa-se diferença significativa para ocorrência de plântulas de G. pohliana, de jovens de E. edulis e G. gamiova e adultos de E. edulis, segundo o teste de Kruskal-Wallis ( $<<0,05$, Tabela 2$)$.

O conhecimento do tamanho e da forma como se encontram estruturadas as populações de plantas de uma determinada área é importante para ações conservacionistas que visam o manejo de espécies (Silvertown e Doust, 1993), bem como para determinar como as diferentes fases da história de vida dessas plantas utilizam os recursos do meio (Svenning, 1999). Mudanças nas relações espécie-ambiente com a ontogenia foram observadas para palmeiras das montanhas andinas (Svenning, 2001). Nas duas parcelas de nosso estudo as plântulas representaram a maioria dos indivíduos da população de palmeiras. Porém, foram os indivíduos jovens e adultos que apresentaram maiores diferenças em número de indivíduos quando comparadas as duas parcelas. Esta diferença pode ter sido ocasionada por algum fator do meio que desencadeou maior mortalidade de plantas na parcela B em relação a A, provavelmente na transição entre os estádios plântula e juvenil.

Quando comparadas as parcelas observou-se que o micro-relevo não influenciou significativamente a distribuição das palmeiras. Apenas as subparcelas cumes para adultos da espécie E. edulis e as vertentes para plântulas de G. gamiova apresentaram diferenças significativas, segundo o teste de Mann-Whitney (Tabela 3). A espécie G. pohliana por não ter sido estatisticamente significativa não foi apresentada na Tabela 3 e G. schottiana não foi considerada para o teste por estar presente somente na parcela A.

O efeito da microtopografia na comunidade de palmeiras foi mais bem percebido quando se comparou o cume das parcelas, que favoreceu principalmente a abundância de adultos da espécie E. edulis na parcela A (Tabela 3). Padrão semelhante foi encontrado na floresta Amazônica Peruana, onde as palmeiras tiveram maior abundância nos cumes (Vormisto et al., 2004). Na floresta montana do presente estudo, a vertente exerceu maior influência em plântulas da espécie G. gamiova. Essa palmeira de subosque pode ter sido prejudicada pela maior declividade que favorece mais a penetração de luz na vertente que nas demais classes topográficas. São vários trabalhos que relacionam à distribuição de palmeiras e as posições topográficas, demonstrando que topografia do local tem forte influência na distribuição das espécies (Kahn e Castro, 1985; Kahn, 1987; Svenning, 1999). De forma contrária, as palmeiras da Costa Rica de grande porte são mais abundantes em declives mais acentuados onde ocorreu abertura de clareiras recentes (Clark et al., 1999). 
Tabela 2. Distribuição do número de indivíduos por estádio ontogenético e comparação entre os valores médios dos estádios das populações de palmeiras amostradas em 0,3 ha nas parcelas A e B na floresta montana do Núcleo Santa Virgínia-SP, segundo o Teste Kruskal-Wallis $\mathrm{P}<0,05$.

\begin{tabular}{ccccccc}
\hline Espécie & Estádio & \multicolumn{2}{c}{ Parcela $\left(\mathrm{N}\right.$ ind $\left.0,3 \mathrm{ha}^{-1}\right)$} & \multicolumn{3}{c}{ Teste de Kruskal-Wallis } \\
& & $\mathrm{A}$ & $\mathrm{B}$ & $\mathrm{H}$ & $\mathrm{P}$ & \\
\hline \multirow{2}{*}{ E. edulis } & plântulas & 1064 & 1103 & 1,63 & 0,2 & $\mathrm{~ns}$ \\
& jovens & 91 & 64 & 3,87 & 0,05 & $*$ \\
& adultos & 55 & 36 & 6,53 & 0,01 & $* *$ \\
& total & $\mathbf{1 2 1 0}$ & $\mathbf{1 2 0 3}$ & & & \\
\hline \multirow{2}{*}{ G. gamiova } & plântulas & 148 & 197 & 3,21 & 0,07 & $\mathrm{~ns}$ \\
& jovens & 129 & 70 & 3,96 & 0,04 & $*$ \\
& adultos & 27 & 38 & 1,72 & 0,18 & $\mathrm{~ns}$ \\
& total & $\mathbf{3 0 4}$ & $\mathbf{3 0 5}$ & & & \\
\hline \multirow{2}{*}{ G. pohliana } & plântulas & 6 & 128 & 3,98 & 0,04 & $*$ \\
& jovens & 0 & 43 & -- & -- & -- \\
& adultos & 0 & 16 & -- & -- & -- \\
& total & 6 & 187 & & & \\
\hline G. schottiana & plântulas & 1 & 0 & -- & -- & -- \\
& jovens & 5 & 0 & -- & -- & -- \\
& adultos & 0 & 0 & -- & -- & -- \\
& total & $\mathbf{6}$ & $\mathbf{0}$ & & & \\
\hline
\end{tabular}

Nota: -- indica que a variável não apresentou número de indivíduos suficiente para análise estatística. ns: não significativo, * significativo em $\mathrm{P}<0,05$; ** significativo em $\mathrm{P}<0,01$.

Tabela 3. Comparação entre os valores médios do número de indivíduos de palmeiras das parcelas A e B em diferentes classes topográficas na floresta montana do Núcleo Santa Virgínia-SP, segundo o Teste Mann-Whitney $(\mathrm{p}<0,05)$.

\begin{tabular}{|c|c|c|c|c|c|c|c|c|c|c|c|c|}
\hline & \multicolumn{4}{|c|}{ Cume ( $\mathrm{N}$ ind $\mathrm{ha}^{-1}$ ) } & \multicolumn{4}{|c|}{ Vertente ( $\mathrm{N}$ ind $\mathrm{ha}^{-1}$ ) } & \multicolumn{4}{|c|}{ Vale $\left(\mathrm{N}\right.$ ind $\left.\mathrm{ha}^{-1}\right)$} \\
\hline & A & B & $\mathrm{P}$ & & A & B & $\mathrm{P}$ & & A & B & $\mathrm{P}$ & \\
\hline \multicolumn{13}{|l|}{ E. edulis } \\
\hline plântulas & 3257 & 1963 & 0,642 & ns & 4100 & 3987 & 0,295 & ns & 3275 & 4350 & 0,343 & ns \\
\hline jovens & 229 & 63 & 0,120 & ns & 293 & 200 & 0,215 & ns & 338 & 263 & 0,558 & ns \\
\hline adultos & 414 & 63 & 0,003 & $* *$ & 200 & 100 & 0,081 & ns & 100 & 138 & 0,350 & ns \\
\hline \multicolumn{13}{|l|}{ G. gamiova } \\
\hline plântulas & 443 & 675 & 0,320 & ns & 350 & 573 & 0,003 & $* *$ & 850 & 500 & 0,916 & ns \\
\hline jovens & 429 & 138 & 0,139 & ns & 521 & 253 & 0,113 & ns & 338 & 275 & 0,874 & ns \\
\hline adultos & 100 & 75 & 0,342 & ns & 71 & 147 & 0,131 & ns & 113 & 125 & 0,955 & ns \\
\hline
\end{tabular}

Nota: ns: não significativo, * significativo em $\mathrm{p}<0,05$; ** significativo em $\mathrm{p}<0,01$.

\subsection{Palmeiras e variáveis edafo-topográficas}

As análises de correlação de Spearman foram usadas para testar as quatro espécies de palmeiras, os três estádios ontogenéticos e dezesseis variáveis ambientais. Nos cumes das parcelas foram obtidas correlações negativas entre potássio e o estádio plântula da palmeira $G$. 
gamiova $\left(\mathrm{r}_{\mathrm{s}}=-0,97, \mathrm{p}=0,003\right)$, positivas entre potássio e jovens de $G$. schottiana $\left(\mathrm{r}_{\mathrm{s}}=0,85\right.$; $\mathrm{p}=0,033)$ e entre alumínio e adultos de G. gamiova $\left(\mathrm{r}_{\mathrm{s}}=0,97, \mathrm{p}=0,003\right)$. Nas vertentes plântulas E. edulis $\left(\mathrm{r}_{\mathrm{s}}=0,84, \mathrm{p}=0,033\right)$ e $G$. pohliana $\left(\mathrm{r}_{\mathrm{s}}=1, \mathrm{p}=0,003\right)$ se correlacionaram positivamente com V\%, e indivíduos jovens de $G$. gamiova $\left(\mathrm{r}_{\mathrm{s}}=0,90\right.$, $\left.\mathrm{p}=0,033\right)$ com a concentração de matéria orgânica - MO. O vale foi a classe topográfica que mais teve correlação com a ocorrência das palmeiras e as variáveis ambientais. Nos vales as plântulas de G. gamiova tiveram correlação positiva com a serapilheira ( $\left.\mathrm{r}_{\mathrm{s}}=0,88, \mathrm{p}=0,033\right)$, os jovens com a fração areia $\left(\mathrm{r}_{\mathrm{s}}=0,98, \mathrm{p}=0,003\right)$ e os adultos correlacionaram-se negativamente com Ca ( $\left.\mathrm{r}_{\mathrm{s}}=-0,86, \mathrm{p}=0,033\right)$. As plântulas $G$. pohliana correlacionaram positivamente com $\mathrm{V} \%\left(\mathrm{r}_{\mathrm{s}}=1, \mathrm{p}=0,003\right)$ e com a soma de bases $\left(\mathrm{r}_{\mathrm{s}}=0,84, \mathrm{p}=0,033\right)$ e as de $E$. edulis com V\% $\left(\mathrm{r}_{\mathrm{s}}=0,84, \mathrm{p}=0,033\right)$. Os estádios plântulas e adultos de $G$. schottiana nos vales, não foram considerados nas análises devido ao baixo número amostral.

Quantos aos resultados da regressão múltipla observou-se que para E. edulis os principais fatores determinantes na distribuição dos adultos foram classes topográficas e umidade. Para G. gamiova os fatores que contribuíram significativamente na distribuição foram classes topográficas, serapilheira e umidade (Tabela 4).

Tabela 4. Modelo resultante da Regressão múltipla entre as palmeiras Euterpe edulis e Geonoma gamiova e variáveis ambientais.

\begin{tabular}{|c|c|c|c|c|c|c|c|}
\hline \multicolumn{8}{|c|}{$\begin{array}{c}\text { Modelo } \\
\text { E. } \text { edulis }=41.422-8.566 * \text { Classes }+0.721 * \text { Umidade }\end{array}$} \\
\hline Fonte & GL & Soma dos quadrados & Média dos quadrados & $\mathrm{F}$ & $\operatorname{Pr}>F$ & AIC & $\mathbf{R}^{2}$ \\
\hline Intercepto & $\begin{array}{c}2 \\
\text { Valor } \\
41.423\end{array}$ & $\begin{array}{c}24330.122 \\
\text { Erro padrão } \\
11.984\end{array}$ & $\begin{array}{c}12165.061 \\
t \\
3.456\end{array}$ & $\begin{array}{c}8.123 \\
\operatorname{Pr}>|t| \\
\mathbf{0 . 0 0 1}\end{array}$ & 0.000 & $\mathbf{8 8 0 , 3 5}$ & 0,12 \\
\hline Classes Topográficas & -8.566 & 2.754 & -3.111 & 0.002 & & & \\
\hline Umidade & 0.721 & 0.318 & 2.265 & 0.025 & & & \\
\hline \multicolumn{8}{|c|}{$\begin{array}{c}\text { Modelo } \\
\text { G. gamiova }=21.871-1.589 * \text { Classes-0.1282*Umidade-1.667 } * \text { Serapilheira }\end{array}$} \\
\hline Fonte & GL & Soma dos quadrados & Média dos quadrados & $\mathrm{F}$ & $\operatorname{Pr}>F$ & AIC & $\mathbf{R}^{2}$ \\
\hline Modelo & $\begin{array}{c}3 \\
\text { Valor }\end{array}$ & $\begin{array}{l}1558.826 \\
\text { Erro padrão }\end{array}$ & $\begin{array}{c}519.609 \\
t\end{array}$ & $\begin{array}{l}6.390 \\
\operatorname{Pr}>|t|\end{array}$ & 0.000 & 530,73 & 0,14 \\
\hline Intercepto & 21.871 & 2.975 & 7.352 & $<0.0001$ & & & \\
\hline Classes Topográficas & -1.590 & 0.655 & -2.426 & 0.017 & & & \\
\hline $\begin{array}{l}\text { Umidade } \\
\text { Serapilheira }\end{array}$ & $\begin{array}{l}-0.128 \\
-1.667 \\
\end{array}$ & $\begin{array}{l}0.075 \\
0.631\end{array}$ & $\begin{array}{l}-1.719 \\
-2.641 \\
\end{array}$ & $\begin{array}{l}0.088 \\
\mathbf{0 . 0 0 9}\end{array}$ & & & \\
\hline
\end{tabular}

Os resultados revelam que as variações ambientais ao longo do gradiente microtopográfico influenciam a distribuição das espécies de palmeiras. A classe vale foi a que mais apresentou influência entre a comunidade de palmeiras e entre as variáveis ambientais, em especial com o gênero Geonoma, em função de possuir menor fertilidade condicionada pelas ações do processo erosivo. Nas florestas subtropicais de Taiwan, Zueng-Sang et al. (1997) consideraram a topografia como responsável pelas modificações nas propriedades de solo, em que as perdas de nutrientes ocorriam principalmente nas regiões de vertentes. Para Vormisto et al. (2004), a topografia não exerce influência direta nas palmeiras amazônicas, mas age indiretamente devido sua relação com outras variáveis ambientais.

Nas parcelas estudadas a fração areia foi a que mais apresentou diferenças significativas com as classes topográficas e a comunidade de palmeiras. Em função do relevo acidentado das áreas a declividade favoreceu o processo erosivo facilitando o transporte e o acúmulo da fração 
areia. Em estudos com palmeiras de trechos da Amazônia peruana (Vormisto et al., 2004) e brasileira (interflúvio Purus-Madeira, Sousa 2007), silte foi a fração dominante nos solos dos locais estudados e a que mais esteve relacionada com variação da composição quantitativa das palmeiras.

Apesar de as parcelas diferirem entre si em relação à umidade do solo nas classes cume e vertente, no presente estudo não se observou influência dessa variável na ocorrência das palmeiras. Provavelmente em função das condições de clima, especificamente da precipitação ser homogênea na área em estudo, a umidade do solo não afetou a comunidade de palmeiras. Em áreas de várzea baixa da floresta Amazônica Jardim et al. (2007) encontraram palmeiras adaptadas a condições de solos férteis e úmidos e observaram que Euterpe oleracea Mart. e Geonoma sp situavam-se entre as de maior abundância. Estudos realizados com a palmeira de dossel Euterpe edulis (Silva Matos e Watikinson, 1998) e com a de subosque Geonoma brevispatha Barb. Rodr. (Souza e Martins, 2004), consideraram-nas como comuns em solos úmidos, como em margens de rios e brejos. A despeito dessa tendência, em nível de microhabitat, Silva Matos e Alves (2008) não encontraram preferência de Geonoma por habitats úmidos, observando sua ocorrência tanto em solos bem drenados como mal drenados. Normand et al. (2006) destacaram que em escala local a umidade do solo é o mais importante controle da diversidade beta de palmeiras em terraços paleo-ribeirinhos da Amazônia peruana. Mesmo sendo baixo o valor da umidade dos solos coletados durante a estação seca, o ambiente montano aqui estudado se mostrou propício à ocorrência de palmeiras desses gêneros devido às condições de fertilidade do solo e da maior umidade relativa do ar, condicionada tanto pela topografia e como pelo microclima do local.

A espessura da camada de serapilheira, que no vale teve seus menores valores, foi positivamente correlacionada com plântulas de G. gamiova. A camada de serapilheira pode influenciar a ocorrência de palmeiras tanto favorável como desfavoravelmente. A barreira física gerada pela presença da serapilheira pode permitir o escape de sementes e plântulas da ação de predadores, como também propiciar condições micro-climáticas para o processo germinativo, estabelecimento e sobrevivência de plântulas (Vásquez-Yanes et al., 1990; Cintra, 1997). Por outro lado, plântulas de palmeiras com sementes pequenas, como as de subosque (p.ex. Geonoma), podem ter dificuldade em penetrar as densas camadas de folhas, serem susceptíveis ao soterramento e, portanto, terem seu recrutamento limitado pela serapilheira (Svenning, 2001). Essa última observação pode explicar a preferência de plântulas de G. gamiova por locais onde a camada de serapilheira apresentou menor espessura. Sousa (2007) verificou também que a composição da comunidade de palmeiras foi afetada pelos efeitos da espessura da serapilheira, encontrando palmeiras de maior porte em locais de serapilheira mais espessa e as de menor porte onde a camada foi menos espessa. No presente estudo indivíduos adultos de E. edulis apresentaram maior ocorrência das parcelas de cume onde a camada de serapilheira foi mais espessa.

Em relação à fertilidade do solo, de forma análoga, o vale foi a classe topográfica que mais influenciou a comunidade de palmeiras. De uma forma geral, de acordo com o estádio ontogenético as palmeiras apresentaram diferentes preferências em relação aos atributos químicos do solo. Nogueira Jr. et al. (2003), observaram que o platô foi a classe que menos favoreceu a sobrevivência de plântulas de E. edulis, enquanto que para indivíduos jovens a sobrevivência foi favorecida na meia encosta e platô.

Na floresta montana em estudo, a concentração de $\mathrm{K}^{+}$nos cumes influenciou a ocorrência de plântulas de G. gamiova e de jovens de G. schottiana. Em uma floresta semidecídua altomontana, em Carrancas-MG, a 1.500 m de altitude, Oliveira Filho et al. (2004) verificaram que a espécie $G$. schottiana teve preferência por solos com menores concentrações de $\mathrm{K}^{+}$. Em levantamento realizado nas proximidades da área do presente estudo, ao longo do gradiente altitudinal da Serra do Mar até 850m, Toledo e Fisch (2006) não observaram a presença $G$. 
schottiana. Pode ser que essa espécie seja restrita a altitudes mais altas da floresta montana da região estudada, porém a forma como o elemento $\mathrm{K}^{+}$influencia a sua ocorrência precisa ser melhor investigada.

Outros elementos químicos também influenciaram a ocorrência de palmeiras em determinados estádios ontogenéticos. Nas vertentes o estádio ontogenético jovem de $G$. gamiova, teve preferência por uma condição intermediária de matéria orgânica - MO, que segundo Silver et al. (1994), tem uma associação com os níveis de P-disponível e $\mathrm{K}^{+}$que favorecem a disponibilidade desses nutrientes para as plantas. A baixa quantidade de indivíduos adultos de G. gamiova teve correlação positiva com os baixos teores de Al nos cumes. Não foi observada a influência do $\mathrm{Al}$ em plântulas e jovens. No entanto, a redução do número de indivíduos com a ontogenia, permitiu que na fase adulta fosse evidenciado que essa espécie é tolerante ao alumínio e consequentemente à acidez do solo. Em geral a toxicidade do $\mathrm{Al}^{+3}$ afeta o desenvolvimento da raiz da planta (Bruijnzeel e Veneklaas, 1998). As palmeiras possuem um sistema radicular superficial e no caso de G. gamiova, além das raízes superficiais, observamse raízes adventícias nos adultos servindo de raízes-escoras. Esse atributo morfológico provavelmente não permite que essa toxicidade interfira no desenvolvimento radicular. Dessa forma, possibilita a ocorrência de maior quantidade de adultos em locais com maiores teores de alumínio, onde as demais espécies são prejudicadas.

Em uma condição de solo com elevada acidez e baixa fertilidade, pode-se afirmar que as palmeiras no presente estudo estão de certa forma adaptadas a esse tipo de ambiente típico da montanha nebular. Sua elevada abundância nessas florestas representa um potencial para a utilização dessas espécies em projetos de recuperação de áreas degradadas (Martins et al. 2003), principalmente em encostas e topos de morro, situação muito comum na Mata Atlântica degradada. As palmeiras além de serem bem representadas na maioria dos ambientes podem ser consideradas como boas indicadoras do seu status de conservação.

\section{CONCLUSÃO}

As diferenças de distribuição de palmeiras na Floresta Ombrófila Densa Montana no Núcleo Santa Virgínia encontradas sugerem que sua composição é determinada pelos requerimentos da própria espécie e sua localização espacial. Das quatro espécies de palmeiras ocorrentes, a mais abundante, Euterpe edulis, parece não apresentar restrições fisiológicas ou mesmo habitats preferenciais para sua ocorrência no ambiente estudado. No entanto, a heterogeneidade microambiental, como a topografia e as propriedades químicas e físicas do solo, ocasionou variação na distribuição e composição local das espécies de palmeiras, em especial do gênero Geonoma.

A despeito da baixa umidade do solo presente nas parcelas durante a estação seca na floresta montana estudada, não se observou influência desse fator com a comunidade de palmeiras. A classe microtopográfica vale foi a que mais apresentou correlações com as variáveis ambientais e com as palmeiras. Ao lado disso, as variáveis edafo-topográficas influenciaram a abundância de determinados estádios ontogenéticos das palmeiras. Entre essas as variáveis que mais se destacaram foram espessura da camada de serapilheira e concentração de areia, que influenciaram a ocorrência de plântulas e jovens de G. gamiova, respectivamente.

A topografia em si não foi o fator delimitador da ocorrência das palmeiras, mas, sim um influenciador da heterogeneidade ambiental na floresta montana.

A elevada densidade das espécies de palmeiras estudadas, em especial a de grande porte Euterpe edulis e a de subosque Geonoma gamiova, indicam que as mesmas estão adaptadas a condições de alta acidez, baixa fertilidade e a umidade do solo da floresta montana nebular. 


\section{AGRADECIMENTOS}

Agradecemos ao apoio financeiro da FAPESP através do Projeto Temático Biota Gradiente Funcional (processo 03/12595-7), nas pessoas de seus coordenadores Profs. Drs. C.A. Joly e L.A. Martinelli; ao apoio logístico do Instituto Florestal/Núcleo Santa Virgínia; ao Professor Serafim Daniel Ballestero pela colaboração na revisão do documento final, após falecimento da primeira autora; a toda equipe de campo do grupo de Ecossistemas Terrestres do Vale do Paraíba e Litoral Norte Paulista. A presente pesquisa foi autorizada pela COTEC/IF 41.424/2006.

\section{REFERÊNCIAS}

BASTOS NETO, A. T.; FISCH, S. T. V. Comunidade de palmeiras no entorno de escorregamentos no Parque Estadual da Serra do Mar- Núcleo Santa Virgínia, SP. Revista Ambiente e Água, v. 2, n. 2, p. 21-32, 2007. http://dx.doi.org/10.4136/1980993X

BROTEL, R. T.; OLIVEIRA FILHO, A. T.; RODRIGUES, L. A.; CURI, N. Influência do solo e topografia sobre variações da composição florística e estrutura da comunidade arbóreoarbustiva de uma floresta estacional semidecidual em Ingaí, MG. Brazilian Journal of Botany, v. 25, n. 2, p. 195-213, 2002. http://dx.doi.org/10.1590/S010084042002000200008

BRUIJNZEEL, L. A.; VENEKLAAS, E. J. Climatic conditions and Tropical Montane Forest productivity: the fog has not lifted yet. Ecology, v. 79, n. 1, p. 3-9, 1998. http://dx.doi.org/10.1890/0012-9658(1998)079[0003:CCATMF]2.0.CO;2

CARVAlHO, D. A.; OLIVEIRA-FILHO, A. T.; VILELA, E. A.; CURI, N.; VAN DEN BERG, E. et al. Distribuição de espécies arbóreo-arbustivas ao longo de um gradiente de solos e topografia em um trecho de floresta ripária do Rio São Francisco em Três Marias, MG, Brasil. Brazilian Journal of Botany, v. 28, n. 2, p. 329-345, 2005. http://dx.doi.org/10.1590/S0100-84042005000200013

CINTRA, R. Leaf litter effects on seed and seedling predation of the palm Astrocaryum murumuru and the legume tree Dipteryx micrantha in Amazonian forest. Journal of Tropical Ecology, v. 13, p. 709-725, 1997. https://doi.org/10.1017/S0266467400010889

CINTRA, R.; XIMENES, A. C.; GONDIM, F. R.; KROPF, M. S. 2005. Forest spatial heterogeneity and palm richness, abundance and community composition in Terra Firme forest, Central Amazon. Brazilian Journal of Botany, v. 28, n. 1, p. 75-84, 2005. http://dx.doi.org/10.1590/S0100-84042005000100007

CLARK, D. B.; PALMER, M. W.; CLARK, D. A. Edaphic factors and the landscape-scale distributions of tropical rain forest trees. Ecology, v. 80, p. 2662-2675, 1999. http://dx.doi.org/10.1890/0012-9658(1999)080[2662:EFATLS]2.0.CO;2

EISERHARDT, W. L.; SVENNING, J. C.; KISSILING, W. D.; BALSLEV, H. Geographical ecology of the palms (Arecaceae): determinants of diversity and distributions across spatial scales. Annals of Botany, p. 1-26, 2011. http://dx.doi.org/10.1093/aob/mcr146

EMBRAPA. Centro Nacional de Pesquisa de Solos. Sistema brasileiro de classificação de solos. Rio de Janeiro 2009. 412p. 
EMBRAPA. Centro Nacional de Pesquisa de Solos.Manual de métodos de análise de solo. 2. ed. Rio de Janeiro, 1997. 212 p.

GENTRY, A. H. Tree species richness of upper Amazonian forests. Proceendigs of the National Academy of Sciences of the United States of América, v. 85, p. 156-159, 1988.

GOLLEY, F. B. Decomposition. In: GOLLEY, F.B. (Ed.). Tropical rain forest ecosystems. Holanda: Elsevier, 1983. p. 157-166.

GRUBB, P. J. Control of Forest growth and distribution on wet tropical mountains: With special reference to mineral nutrition. Annual Review of Ecology and Systematics, v. 8, p. 83107, 1977. http://www.jstor.org/stable/2096722

JARDIM, M. A. G.; SANTOS, G. C.; MEDEIROS, T. D. S. ; FRANCEZ, D. C. Diversidade e estrutura da palmeiras em Floresta de Várzea do Estuário Amazônico. Amazônia: Ciência \& Desenvolvimento, v. 2, n. 4, p. 67-84, 2007.

JOLY, C. A.; ASSIS, M. A.; BERNACCI, L. C.; TAMASHIRO, J. Y.; CAMPOS, M. C. R.; GOMES, J. A. M. A. et al. Floristic and phytosociology in permanent plots of the Atlantic Rainforest along an altitudinal gradient in southeastern Brazil. Biota Neotropica, v. 12, n. 1, 2012. http://dx.doi.org/10.1590/S1676-06032012000100012

KAHN, F.; CASTRO, A. The Palm community in a forest of central amazonia, Brazil. Biotropica, v. 17, n. 3, p. 210-216, 1985. http://dx.doi.org/10.2307/2388221

KAHN, F. The distribuition of palms as a function of local topography in Amazonian terra$\begin{array}{llllll}\text { firme forests. } & \text { Experientia, } & \text { v. 43, } & \text { p. 251-259, }\end{array}$ http://dx.doi.org/10.1007/BF01945548

KAPPELLE, M.; GEUZE, T.; LEAL, M.; CLEF, M. Successional age and forest structure in a Costa Rica upper montane Quercus forest. Journal of Tropical Ecology, v. 12, n. 681698, 1996. https://doi.org/10.1017/S0266467400009871

MARTINS, S. V.; SILVA, N. R. S.; SOUZA, A. L.; MEIRA NETO, J. A. A. Distribuição de espécies arbóreas em um gradiente topográfico de Floresta Estacional Semidecidual em Viçosa, MG. Scientia Florestalis, v. 64, p. 172-181, 2003.

MORELlATO, L. P. C.; HADDAD, C. F. B. Introduction: the brazilian atlantic forest. Biotropica, v. 32, p.786-792, 2000. http://dx.doi.org/10.1111/j.17447429.2000.tb00618.x

NOGUEIRA JR., L. R.; FISCH, S. T. V.; BALLESTERO, S. D. Influência da umidade do solo no desenvolvimento inicial de plantas do palmiteiro Euterpe edulis Mart. em Floresta Nativa. Revista Biociências, v. 9, n. 1, p. 7-13, 2003.

NORMAND, S.; VORMISTO, J.; SVENNING, J.; GRÁNDEZ, C.; BALSLEV, H. Geographical and environmental controls of palm beta diversity in paleo-riverine terrace forests in Amazonian Peru. Plant Ecology, v. 186, p. 161-176, 2006. http://dx.doi.org/10.1007/s11258-006-9120-9

OLIVEIRA FILHO, A. T.; CARVALHO, D. A.; FONTES, M. A. L.; VAN DEN BERG, E.; CURI, N.; CARVALHO, W. A. C. Variações estruturais do compartimento arbóreo de uma floresta semidecídua alto-montana na chapada das Perdizes, Carrancas, MG. Brazilian Journal of Botany, v. 27, n. 2, p. 291-309, 2004. http://dx.doi.org/10.1590/S0100-84042004000200009 
OLIVEIRA, K. F.; FISCH, S. T. V.; DUARTE, J.; DANELLI, M.F.; MARTINS, L. F. S.; JOLY, C. Estrutura e distribuição espacial de populações de palmeiras em diferentes altitudes na Serra do Mar, Ubatuba-SP. Rodriguésia, v. 65, p. 1043-1055, 2014. http://dx.doi.org/10.1590/2175-7860201465414

PORTELA, R. C. Q.; SANTOS, F. A. M. Produção e espessura da serapilheira na borda e interior de fragmentos florestais de Mata Atlântica de diferentes tamanhos. Brazilian Journal of Botany, v. 30, n. 2, p. 271-280, 2007. http://dx.doi.org/10.1590/S010084042007000200011

RAIJ, B. V.; CANTARELlA, H.; QUAGGIO, J. A. ; FURLANI, A. M. C. (Eds.) Recomendações de adubação e calagem para o Estado de São Paulo. 2. ed. Campinas: Instituto Agronômico; Fundação IAC, 1996. 285 p. (Boletim Técnico, 100).

RESENDE, M.; LANI, J. L.; REZENDE, S. B. Pedossistemas da Mata Atlântica: considerações pertinentes sobre a sustentabilidade. Revista Árvore, v. 26, n. 3, p. 261-269, 2002. http://dx.doi.org/10.1590/S0100-67622002000300001

RICHARDS, P. W. The tropical rain forest. 2. ed. Cambridge: Cambridge University Press, 1996. 575 p.

RODRIGUES, L. A.; CARVALHO, D. A.; OLIVEIRA-FILHO, A. T.; CURI, N. Efeitos de solos e topografia sobre a distribuição de espécies arbóreas em um fragmento de floresta estacional semidecidual, em Luminárias, MG. Revista Árvore, v. 31, n. 1, p. 25-35, 2007. http://dx.doi.org/10.1590/S0100-67622007000100004

ROSS, S. M. Soil and vegetation effects of tropical deforestation. In: GOLDSMITH, F. B. (Ed.). Tropical rain forest: a wider perspective. Londres: Chapman \& Hall, 1998.

SCARANO, F. R. Structure, function and floristic relantioships of plants communities in stressful habitats marginal to Brazilian Atlantic Rainforest. Annals of Botany, v. 90, p. 517-524, 2002. http://dx.doi.org/10.1093/aob/mcf189

SCHLITTLER, F. H. M.; DE MARINES,G.; CESAR, O. Produção de serapilheira na floresta do morreo do diabo, Pontal do Paranapanema-SP. Naturalia, v. 18, p.135-147, 1993.

SCUDELLER, V. V.; MARTINS, F. R.; SHEPHERD, G. J. Distribution and abundance of arboreal species in the atlantic ombrophilous dense forest in Southeastern Brazil. Plant Ecology, v. 152, p. 185-199, 2001. http://dx.doi.org/10.1023/A:1011494228661

SILVA MATOS, D. M. S.; WATKINSON, A. R. The fecundity, seed and seedling ecology of the edible palm Euterpe edulis in Southeastern Brazil. Biotropica, v. 30, n. 4, p. 595-603, 1998. http://dx.doi.org/10.1111/j.1744-7429.1998.tb00099.x

SILVA, M. D. G. P. C.; MARTINI, A. M. Z. ; ARAÚJO, Q. R. Estrutura populacional de Euterpe edulis Mart. no Sul da Bahia , Brasil. Brazilian Journal of Botany, v. 32, n. 2, p. 393-403, 2009. http://dx.doi.org/10.1590/S0100-84042009000200017

SILVA MATOS, D. M. S. ; ALVES, L.F. Palm species distribution and soil moisture in a swampy area of the Atlantic Forest, South-eastern Brazil. Ecotropica, v. 14, p. 69-74, 2008. 
SILVER, W. L.; SCATENA, F. N.; JOHNSON, A. H.; SICCAMA, T. G. ; SANCHEZ, M. J. Nutrient availability in a montane wet tropical forest: Spatial patterns and methodological considerations. Plant and Soil, v. 164, p. 129-145, 1994.

http://dx.doi.org/10.1007/BF00010118

SILVERTOWN, J. W.; DOUST, J. L. Intraspecific interactions. In: SILVERTOWN, J.; CHARLESWORTH, D. Introduction to plant population biology. Oxford: Blackwell, 1993. p.51-57.

SOUSA, T. E. L. Distribuição de Palmeiras (Arecaceae) ao longo de gradientes ambientais no baixo interflúvio Purus-Madeira, Brasil. 2007. 42 p. Dissertação (Mestrado) Universidade Federal do Amazonas, Manaus, 2007.

SOUZA, A. F.; MARTINS, F. R. Microsite specialization and spatial distribution of Geonoma brevispatha, a clonal palm in south-eastern Brazil. Ecological Research, v. 19, p. 521532, 2004. http://dx.doi.org/10.1111/j.1440-1703.2004.00670.x

SOUZA, A. F.; MARTINS, F. R.; BERNACCI, L. C. Clonal growth and reproductive strategies of the understory tropical palm Geonoma brevispatha: an ontogenetic approach. Canadian Journal of Botany, v. 81, p. 101-112, 2003. http://dx.doi.org/10.1139/B03002

SVENNING, J. C. Microhabitat specialization in a species-rich palm community in Amazonian Ecuador. Journal of Ecology, v. 87, p. 55-65, 1999. http://dx.doi.org/10.1046/j.13652745.1999.00329.x

SVENNING, J. C. On the role of microenvironmental heterogeneity in the ecology and diversification on neotropical rain-forest palms (Arecaceae). The Botanical Review, v. 67, p. 1- 53, 2001. http://dx.doi.org/10.1007/BF02857848

SVENNING, J. C.; HARLEV, D.; SORENSEN, M. M.; BALSLEV, H. Topographic and spatial controls of palm species distributions in a montane rain forest, southern Ecuador. Biodivers Conserv, v. 18, p. 219-228, 2009. http://dx.doi.org/10.1007/s10531-0089468-3

TOLEDO, M. C. B.; FISCH, S.T.V. Bases cartográficas para armazenamento e análise espacial de dados da diversidade de palmeiras em um trecho da Mata Atlântica, Ubatuba-SP. Biota Neotropica, v. 6, n. 1, 2006.

TOMLINSON, P. B. The uniqueness of palms. Botanical Journal of the Linnean Society, v. 151, p. 5-14, 2006. http://dx.doi.org/10.1111/j.1095-8339.2006.00520.x

VASHCHENKO, Y.; PIOVESAN, R. P.; LIMA, M. R.; FAVARETTO, N. Solos e vegetação dos picos Camacuã, Camapuã e Tucum - Campina Grande do Sul - PR. Scientia Agraria, v. 8, n. 4, p. 411-419, 2007.

VÁSQUEZ-YANES, C.; OROZCO-SEGOVIA, A.; RINCÓN, E.; SÁNCHEZ-CORONADO, M. E.; HUANTE, P.; TOLEDO, J. R. et al. Light beneath litter in a tropical Forest: effect on seed germination. Ecology, v. 71, p. 1952-1958, 1990. http://dx.doi.org/10.2307/1937603

VELOSO, H. P.; RANGEL FILHO, A. L. R.; LIMA, J. C. A. Classificação da vegetação brasileira, adaptada a um sistema universal. Rio de Janeiro: IBGE, 1991. 
VORMISTO, J.; TUOMISTO, H.; OKSANSEN, J. Palm distribution patterns in Amazonian rainforests: what is the role of topographic variation? Journal of Vegetation Science, v. 15, p. 485-494, 2004. http://dx.doi.org/10.1111/j.1654-1103.2004.tb02287.x

WIDYATMOKO, D.; BURGMAN, M. A. Influences of edaphic factors on the distribution and abundance of a rare palm (Cyrtostachys renda) in a peat swamp forest in eastern Sumatra, Indonesia. Austral Ecology, v. 31, p. 964-974, 2006. http://dx.doi.org/10.1111/j.14429993.2006.01672.x

YASUHIRO, K.; HIROFUMI, M.; KIHACHIRO, K. Effects of topographic heterogeneity on tree species richness and stand dynamics in a subtropical forest in Okinawa Island, southern Japan. Journal of Ecology, v. 92, p. 230-240, 2004. http://dx.doi.org/10.1111/j.0022-0477.2004.00875.x

ZAR, J.H. Biostatistical analysis. Englewood Cliffs: Prentice-Hall, 1996, 662p.

ZUENG-SANG, C.; CHANG-FU, H.; FEEI-YU, J.; TSUNG-HSIN, H. ; I-FANG, S. Relations of soil properties to topography and vegetation in a subtropical rain forest in southern Taiwan. Plant Ecology, v. 132, p. 229-241, 1997.

http://dx.doi.org/10.1023/A:1009762704553 